\title{
DETOUR TRANSITIONS IN INTERNAL BREMSSTRAHLUNG
}

\author{
G. W. FORD and C. F. MARTIN \\ Department of Physics, The University of Michigan, Ann Arbor, Michigan, USA ${ }^{\dagger}$
}

Received 6 May 1969

\begin{abstract}
The contribution of so-called detour transitions to the internal bremsstrahlung accompanying nuclear beta decay is calculated. These are transitions in which the nucleus rather than the electrons radiates the photon. It is shown that such contributions will in general be important for forbidden beta decays. For the unique first-forbidden case, the photon spectrum, angular correlation and polarization are calculated explicitly.
\end{abstract}

\section{Introduction}

In recent years, there have been a number of improved measurements of the spectrum and angular correlation of internal bremsstrahlung accompanying nuclear beta decay ${ }^{1-3}$ ). The consensus is that, at least for the forbidden transitions, there is a considerable discrepancy between the experimental results and the predictions of the Knipp-Uhlenbeck-Bloch theory of internal bremsstrahlung ${ }^{4,5}$ ). In general, the observed spectrum is higher than the predicted spectrum, the disparity being greater the higher the photon energy. Inclusion of Coulomb effects in the theoretical calculations reduces the disagreement, but still leaves a large discrepancy ${ }^{6,7}$ ).

In this paper, we consider the effect of so-called "detour transitions" on the spectrum, angular correlation, and polarization of inner bremsstrahlung ${ }^{8-10}$ ). In the language of perturbation theory, detour transitions are those in which the parent nucleus first emits the photon and goes into a virtual intermediate excited state from which it subsequently beta decays to the final state or vice versa. These detour transitions are to be contrasted with the "direct transitions" of the Knipp-UhlenbeckBloch theory in which the photon is emitted by the outgoing electron.

One might think that since the radiation efficiency of a particle is approximately inversely proportional to its mass, the intensity of the radiation from the detour transition would be smaller than that from the direct transition by a factor of the order of the square of the electron-nucleon mass ratio. This is indeed the case for an allowed beta transition, where the radiation from detour transitions is negligible; but for forbidden transitions, the situation is somewhat different. If the direct transition is forbidden, the beta decay in the detour transition can be allowed and, since the ratios of beta-transition matrix elements in the various orders of forbiddenness are roughly proportional to the nucleon mass, the detour transition might be expected

$\dagger$ Work supported in part by the National Science Foundation. 
to compete favorably with the direct decay. Indeed, we shall see that under certain reasonable approximations, exactly the same nuclear matrix elements occur in the direct and detour transition rates for forbidden beta decay. Another effect enhancing the importance of detour transitions stems from the fact that the spectrum of inner bremsstrahlung from the direct transition is of the " $1 / k$ " type, with most of the radiation at low frequencies (familiar in the case of ordinary external bremsstrahlung). The radiation from the detour transition, on the other hand, will be nuclear multipole radiation with most of the radiation at high frequencies. Thus, although the total intensity of radiation from the detour transitions may be small compared with that from the direct transition, the high frequency portion of the spectrum can be dominated by the detour transitions.

\section{The transition rates}

For simplicity we shall calculate the effect of the detour transitions only for the unique first-forbidden beta decay $(\Delta J=2$, yes). We shall later make some remarks upon the effect in other decays. Consider, then, a transition in which the parent nucleus is in a (nuclear) state $a$ which beta decays to the daughter in a state $c$. Since this direct transition is unique first forbidden, the states $a$ and $c$ must have opposite parity

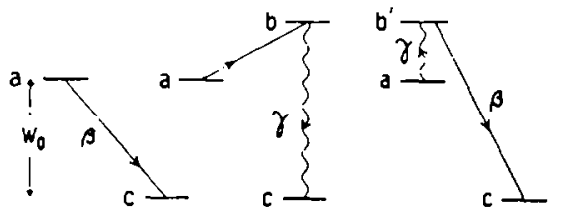

Fig. 1. Direct and detour Transitions.

and have total angular momentum differing by two units. Accompanying this direct decay there will be the usual inner bremsstrahlung emitted by the electron. Coherent with this direct transition will be the detour transition in which the nucleus first beta decays to a virtual excited state $b$ in the daughter nucleus and then emits a photon in an electromagnetic transition from the state $b$ to the final state $c$ in the daughter nucleus. Of course, there can also be the reverse-order process in which the nucleus first emits a photon and then beta-decays (see fig. 1). In the detour transitions, there is no restriction on the energy of the excited nuclear states since we must conserve energy only in the overall transition $a \rightarrow c$. However, if we assume the detour beta decay is allowed $(\Delta J=1$, no) and the electromagnetic transition electric-dipole $(\Delta J=1$, yes), then the spin and parity of the excited state are fixed by the requirement that the overall transition be unique first forbidden $(\Delta J=2$, yes). Other combinations of forbiddenness in the detour beta decay, e.g. first forbidden ( $\Delta J=1$, yes) and magnetic dipole $(\Delta J=1$, no), will clearly give a much smaller contribution to the decay rate and may be neglected. 
In our calculations, we shall use a formalism similar to that used in the original paper of Knipp and Uhlenbeck ${ }^{4}$ ); see also the paper on Coulomb corrections by Lewis and Ford ${ }^{11}$ ). Consider first the ordinary direct beta decay, i.e. the transition without inner bremsstrahlung. The matrix element for this transition is

$$
\left(\mathrm{f}\left|H_{\beta}\right| \mathrm{i}\right)=2^{-\frac{3}{2}} i g \lambda u^{\dagger}(\boldsymbol{p}) N \cdot \sigma\left(1+\gamma_{5}\right) u(q),
$$

where $u(p)$ and $u(q)$ are the plane wave amplitudes for the electron and neutrino, respectively, and the Gamow-Teller coupling constant $g \lambda=1.67 \times 10^{-49} \mathrm{erg} \cdot \mathrm{cm}^{3}$ (the Fermi coupling does not contribute to the unique first-forbidden transition). Also in this expression,

$$
N_{i}=B_{i j}\left(p_{j}+q_{j}\right) \text {, }
$$

where $B_{i j}$ is the nuclear matrix element for the unique forbidden decay. It has the form

$$
B_{i j}=\left\langle c\left|\sum_{n=1}^{A}\left\{\left(\sigma_{i} x_{j}+\sigma_{j} x_{i}-\frac{2}{3} \sigma \cdot r \delta_{i j}\right) \tau_{+}\right\}_{n}\right| a\right\rangle,
$$

where the operators within the braces are the spin, coordinate and isospin of the $n$th nucleon and the states $|a\rangle$ and $|c\rangle$ the initial and final nucleon states, respectively.

The total beta transition rate is

$$
I_{\beta}=2 \pi \sum_{\mathrm{f}}\left|\left(\mathrm{f}\left|H_{\beta}\right| \mathrm{i}\right)\right|^{2} \delta\left(E_{\mathrm{i}}-E_{\mathrm{f}}\right),
$$

where the sum is over all final states of the electron and neutrino. Inserting eq. (2.1), we can write

$$
\begin{aligned}
\Gamma_{\beta}=\frac{g^{2} \lambda^{2}}{8(2 \pi)^{5}} \int \mathrm{d} p \int \mathrm{d} q \delta\left(W+q-W_{0}\right) u^{\dagger}(p) N \cdot \sigma\left(1+\gamma_{5}\right) u(q) & \\
& \times u^{\dagger}(q)\left(1+\gamma_{5}\right) N^{*} \cdot \sigma u(p),
\end{aligned}
$$

where the integrals represent integration over the momenta and summation over the spins of the electron and neutrino and $W_{0}$ is the difference in energy between the initial and final nuclear states. The sum over spins in this expression can be performed using the projection operators

$$
\begin{gathered}
\sum_{\text {spin }} u(q) u^{\dagger}(q)=\frac{q+\alpha \cdot q}{2 q}, \\
\sum_{\text {spin }} u(p) u^{\dagger}(p)=\frac{W+\mathscr{H}(p)}{2 W},
\end{gathered}
$$

where $\mathscr{H}(\boldsymbol{p})=\boldsymbol{\alpha} \cdot \boldsymbol{p}+m \beta$ is the Dirac Hamiltonian. We find

$$
\Gamma_{\beta}=\frac{g^{2} \lambda^{2}}{4(2 \pi)^{5}} \int_{m}^{W_{0}} \mathrm{~d} W p q \int \mathrm{d} \Omega_{\mathrm{e}} \int \mathrm{d} \Omega_{v}\left[N \cdot N^{*}(q W-q \cdot p)+N \cdot q N^{*} \cdot p+N \cdot p N^{*} \cdot q\right]
$$

in which $q=W_{0}-W$. 
The integrals over the directions of the electron and neutrino are straightforward, remembering that $p$ and $q$ occur in $N$ through eq. (2.2) and we obtain the well-known result

$$
\Gamma_{\beta}=\frac{g^{2} \lambda^{2} B_{r s} B_{r s}^{*}}{24 \pi^{3}} \int_{m}^{W o} \mathrm{~d} W W p q^{2}\left(p^{2}+q^{2}\right) .
$$

We now calculate the matrix element for the emission of inner bremsstrahlung in the direct transition, which is of the form

$$
\left(M_{\mathrm{fi}}\right)^{\mathrm{dir}}=\sum_{j} \frac{\left(\mathrm{f}\left|H_{y}\right| j\right)\left(j\left|H_{\beta}\right| \mathrm{i}\right)}{E_{\mathrm{i}}-E_{j}} .
$$

Here the electromagnetic matrix element for the emission of the photon by the electron is

$$
\left(\mathbf{f}\left|H_{j}\right| j\right)=\left(\frac{2 \pi \alpha}{k}\right)^{\frac{1}{2}} u^{\dagger}(\boldsymbol{p}) \hat{e}^{*}+\alpha u\left(p_{1}\right)
$$

where $\hat{e}$ is the photon polarization vector, and the momentum of the intermediate electron is

$$
p_{1}=p+k,
$$

with $\boldsymbol{k}$ the momentum of the outgoing photon. The beta-decay matrix element is

where

$$
\left(j\left|H_{\beta}\right| \mathbf{i}\right)=2^{-\frac{3}{2}} i g \lambda u^{\dagger}\left(p_{1}\right) L \cdot \sigma\left(1+\gamma_{5}\right) u(q),
$$

$$
L_{\mathrm{i}}=B_{\mathrm{ij}}\left(p_{1 j}+q_{j}\right) \text {, }
$$

with $B_{i j}$ the nuclear matrix element eq. (2.3). The energy denominator in eq. (2.9) can be written

$$
\begin{aligned}
\left(E_{\mathrm{i}}-E_{\mathrm{j}}\right)^{-1} & =\left[W_{\mathrm{e}}-\mathscr{H}\left(\boldsymbol{p}_{1}\right)\right]^{-1} \\
& =\frac{\mathscr{H}\left(\boldsymbol{p}_{1}\right)+W_{\mathrm{e}}}{W_{\mathrm{e}}^{2}-W_{1}^{2}},
\end{aligned}
$$

where $W_{\mathrm{e}}=W_{0}-q$, and $\mathscr{H}\left(\boldsymbol{p}_{1}\right)=\alpha \cdot \boldsymbol{p}_{1}+\beta m$ is the Dirac Hamiltonian for the intermediate state electron. Summing over intermediate states we get

$$
\left(M_{\mathrm{fi}}\right)^{\mathrm{dir}}=\frac{1}{2} i g \lambda\left(\frac{\pi \alpha}{k}\right)^{\frac{1}{2}} u^{\dagger}(\boldsymbol{p}) \hat{\boldsymbol{e}}^{*} \cdot \alpha \frac{\mathscr{H}\left(\boldsymbol{p}_{1}\right)+W_{\mathrm{e}}}{W_{\mathrm{e}}^{2}-W_{1}^{2}} \boldsymbol{L} \cdot \sigma\left(1+\gamma_{5}\right) u(\boldsymbol{q}) .
$$

We now consider the detour transitions for which the matrix element is of the form

$$
\left(M_{\mathrm{fi}}\right)^{\text {det }}=\sum_{j} \frac{\left(\mathrm{f}\left|H_{\gamma}\right| j\right)\left(j\left|H_{\beta}\right| \mathrm{i}\right)}{E_{\mathrm{i}}-E_{j}}+\sum_{j} \frac{\left(\mathrm{f}\left|H_{\beta}\right| j\right)\left(j\left|H_{\gamma}\right| \mathrm{i}\right\rangle}{E_{\mathrm{i}}-E_{j}} .
$$

Here the first expression on the right-hand side will correspond to the detour transition in which the nucleus first beta-decays and then radiates the photon; the second 
term corresponds to the processes in reverse order. As we have argued above, if the direct transition is unique first forbidden, the detour beta decay must involve an allowed transition with $\Delta J=1$. This is the selection rule for the so-called unique allowed transition to which only the Gamow-Teller coupling contributes. The matrix element for the beta transition in the first term of eq. (2.16) is

$$
\left(j\left|H_{B}\right| i\right)=-2^{-\frac{1}{2}} g \dot{\lambda}\left\langle b\left|\sum_{n=1}^{A}\left\{\sigma \tau_{+}\right\}_{n}\right| a\right\rangle \cdot u^{\dagger}(p) \sigma\left(1+\gamma_{5}\right) u(q) .
$$

The matrix element for the beta transition in the second term of eq. (2.16) is the same except that in the nuclear matrix element, the states $b$ and a are replaced by the states $c$ and $b^{\prime}$, respectively. The electromagnetic matrix element in the first term of eq. (2.16) is

$$
\left(\mathrm{f}\left|H_{\gamma}\right| j\right)=-\left(\frac{2 \pi \alpha}{k}\right)^{\frac{t}{t}}\left\langle c\left|\sum_{n=1}^{A}\left\{\hat{e}^{*} \cdot \alpha \frac{1+\tau_{3}}{2}\right\}_{n}\right| b\right\rangle,
$$

while that in the second term is the same except for the replacement of states $c$ and $b$ by states $b^{\prime}$ and $a$. Inserting these results and the energy denominators in eq. (2.16), we can write

$$
\left(M_{\mathrm{fi}}\right)^{\mathrm{det}}=\frac{1}{2} i g \lambda\left(\frac{\pi \alpha}{k}\right)^{\frac{1}{2}} u^{\dagger}(p) \boldsymbol{K}^{\prime} \cdot \sigma\left(1+\gamma_{5}\right) u(q)
$$

where

with

$$
K_{j}^{\prime}=B_{j k}^{\prime} \hat{e}_{k}^{*}
$$

$$
\begin{aligned}
B_{j k}^{\prime} & =\sum_{b} \frac{\left\langle c\left|\sum_{n=1}^{A}\left\{-i x_{k}\left(1+\tau_{3}\right)\right\}_{n}\right| b\right\rangle\left\langle b\left|\sum_{m=1}^{A}\left\{\sigma_{j} \tau_{+}\right\}_{m}\right| a\right\rangle}{E_{a}-E_{b}-q-W} \\
& +\sum_{b^{\prime}} \frac{\left\langle c\left|\sum_{m=1}^{A}\left\{\sigma_{j} \tau_{+}\right\}_{m}\right| b^{\prime}\right\rangle\left\langle b^{\prime}\left|\sum_{n=1}^{A}\left\{-i x_{k}\left(1+\tau_{3}\right)\right\}_{n}\right| a\right\rangle}{E_{a}-E_{b^{\prime}}-k}
\end{aligned}
$$

Since the direct and detour transitions are coherent, the total matrix element for the emission of an inner bremsstrahlung photon is

$$
\begin{aligned}
M_{\mathrm{fl}} & =\left(M_{\mathrm{fi}}\right)^{\mathrm{dit}}+\left(M_{\mathrm{fi}}\right)^{\mathrm{det}} \\
& =\frac{1}{2} i q \lambda\left(\begin{array}{c}
\pi \alpha \\
k
\end{array}\right)^{t} u^{\dagger}(\boldsymbol{p})\left[\hat{\boldsymbol{e}}^{*} \cdot \frac{\mathscr{H}\left(\boldsymbol{p}_{1}\right)+W_{\mathrm{c}}}{W_{\mathrm{e}}^{2}-W_{1}^{2}} \boldsymbol{L} \cdot \sigma+\boldsymbol{K}^{\prime} \cdot \sigma\right]\left(1+\gamma_{5}\right) u(\boldsymbol{q}) .
\end{aligned}
$$

The transition rate is

$$
\begin{aligned}
\Gamma_{\gamma} & =2 \pi \sum_{\mathrm{f}}\left|M_{\mathrm{fi}}\right|^{2} \delta\left(E_{\mathrm{i}}-E_{\mathrm{f}}\right) \\
& =\frac{1}{(2 \pi)^{8}} \int \mathrm{d} k \int \mathrm{d} p \int \mathrm{d} q\left|M_{\mathrm{fi}}\right|^{2} \delta\left(W_{0}-W-k-q\right),
\end{aligned}
$$


where the integrals represent integrals over the momenta and summation over the spins of the electron, neutrino and photon. Inserting eq. (2.22) and performing the sum over spins as in the above discussion of $\Gamma_{\beta}$, we can write

$$
\Gamma_{\gamma}=\frac{g^{2} \lambda^{2} \alpha}{4(2 \pi)^{7}} \int_{0}^{W_{0}-m} \mathrm{~d} k \int_{m}^{W_{0}-k} \mathrm{~d} W p W q^{2} k \int \mathrm{d} \Omega_{\gamma} \int \mathrm{d} \Omega_{\mathrm{e}} \int \mathrm{d} \Omega_{v} T,
$$

where $q=W_{0}-W-k$, and where

$$
\begin{aligned}
T=\frac{1}{4 q W} \operatorname{Tr}\left[\left\{\hat{e}^{*} \cdot \alpha \frac{\mathscr{H}\left(\boldsymbol{p}_{1}\right)+W_{\mathrm{e}}}{W_{\mathrm{e}}^{2}-W_{1}^{2}} \boldsymbol{L} \cdot \boldsymbol{\sigma}+\boldsymbol{K}^{\prime} \cdot \boldsymbol{\sigma}\right](q+\boldsymbol{q} \cdot \boldsymbol{\alpha})\left(1+\gamma_{5}\right)\right. \\
\left.\times\left[\boldsymbol{L}^{*} \cdot \boldsymbol{\sigma} \frac{\mathscr{H}\left(\boldsymbol{p}_{1}\right)+W_{\mathrm{e}}}{W_{\mathrm{c}}^{2}-W_{1}^{2}} \hat{e}^{*} \cdot \boldsymbol{\alpha}+\boldsymbol{K}^{\prime *} \cdot \boldsymbol{\sigma}\right][\mathscr{H}(\boldsymbol{p})+W]\right\} .
\end{aligned}
$$

The result of forming the indicated trace is a rather lengthy expression. It becomes more manageable, however, if we average over directions of the neutrino and over nuclear orientations. Denoting this average with a bar, we can write

$$
\begin{aligned}
\bar{T} & =\frac{1}{3} B_{i j} B_{i j}^{*} \frac{p_{1}^{2}+q^{2}}{W\left(W_{\mathrm{e}}^{2}-W_{1}^{2}\right)^{2}}\left\{W\left(W_{\mathrm{e}}^{2}+W_{1}^{2}\right)-2 W_{\mathrm{e}}\left(\hat{\boldsymbol{k}} \cdot \boldsymbol{p} \hat{\boldsymbol{k}} \cdot \boldsymbol{p}_{1}+m^{2}\right)\right. \\
& \left.-2 \varepsilon k\left[W W_{\mathrm{c}}-m^{2}-\hat{\boldsymbol{k}} \cdot \boldsymbol{p} \hat{\boldsymbol{k}} \cdot \boldsymbol{p}_{1}\right]\right\} \\
& +\frac{1}{3} \operatorname{Rl}\left\{B_{i j}^{*} B_{i j}^{\prime}\right\} \frac{1}{W\left(W_{\mathrm{e}}^{2}-W_{1}^{2}\right)}\left\{\left(W_{\mathrm{e}}+W-\frac{1}{2} k\right)\left(p^{2}-\hat{\boldsymbol{k}} \cdot \boldsymbol{p}\right)^{2}-W \hat{k} \cdot \boldsymbol{p}_{1}+k \boldsymbol{p} \cdot \boldsymbol{p}_{1}\right. \\
& \left.-\varepsilon k\left[\frac{1}{2}\left(p^{2}-\hat{k} \cdot \boldsymbol{p}^{2}\right)-W \hat{k} \cdot \boldsymbol{p}_{1}+\hat{\boldsymbol{k}} \cdot \boldsymbol{p} \hat{k} \cdot \boldsymbol{p}_{1}\right]\right\}+\frac{1}{3} B_{i j}^{\prime} B_{i j}^{\prime *}\left\{1-\frac{\varepsilon}{2 W} \hat{k} \cdot \boldsymbol{p}\right\},
\end{aligned}
$$

where $\varepsilon= \pm 1$ for right and left circularly polarized photons, respectively.

Before we proceed further with the discussion of these results, we consider the nuclear matrix elements of the detour transition.

\section{The nuclear matrix elements for the detour transition}

The detour nuclear matrix elements given by eq. (2.21) can with certain assumptions be shown to be identical with the direct matrix elements given by eq. (2.3). The first point is that the Dirac matrix operator occurring in the electromagnetic matrix elements is the velocity operator for the $n$th nucleon and may therefore be expressed in terms of the commutator

$$
-i\{\boldsymbol{\alpha}\}_{n}=\left[\boldsymbol{H},\{\boldsymbol{r}\}_{n}\right]
$$

Here $\boldsymbol{H}$ is the nuclear Hamiltonian and $\{\boldsymbol{r}\}_{n}$ the coordinate operator for the $n$th nucleon. But the nuclear Hamiltonian commutes with $\left\{\tau_{z}\right\}_{n}$ because of the charge independence of the nuclear forces, and the states $|a\rangle,|b\rangle$ etc. occurring in eq. (2.21) 
are eigenstates of $\boldsymbol{H}$ with energies $E_{a}, E_{b}$, etc. Hence, we obtain

$$
\begin{aligned}
\left\langle c\left|\sum_{n=1}^{A}\left\{-i \alpha\left(1+\tau_{3}\right)\right\}_{n}\right| b\right\rangle & =\langle c|\left[H, \sum_{n=1}^{A}\left\{r\left(1+\tau_{3}\right)\right\}_{n}|b\rangle\right. \\
& =\left(E_{c}-E_{b}\right)\left\langle c\left|\sum_{n=1}^{A}\left\{r\left(1+\tau_{3}\right)\right\}_{n}\right| b\right\rangle,
\end{aligned}
$$

and similarly for the corresponding matrix element in the second terms of eq. (2.21). Inserting these expressions in eq. (2.21), we have

$$
\begin{aligned}
B_{j k}^{\prime}=\sum_{b}\left\langle c\left|\sum_{n=1}^{A}\left\{x_{k}\left(1+\tau_{3}\right)\right\}_{n}\right| b\right\rangle \frac{E_{b}-E_{c}}{E_{b}-E_{c}-k}\left\langle b\left|\sum_{m=1}^{A}\left\{\sigma_{j} \tau_{+}\right\}_{m}\right| a\right\rangle \\
-\sum_{b^{\prime}}\left\langle c\left|\sum_{m=1}^{A}\left\{\sigma_{j} \tau_{+}\right\}_{m}\right| b^{\prime}\right\rangle \frac{E_{b^{\prime}}-E_{a}}{E_{b^{\prime}}-E_{a}+k}\left\langle b^{\prime}\left|\sum_{n=1}^{A}\left\{x_{k}\left(1+\tau_{3}\right)\right\}_{n}\right| a\right\rangle .
\end{aligned}
$$

The next point stems from the remark that the states $|b\rangle$ and $\left|b^{\prime}\right\rangle$ are states connected with the daughter or parent nuclear ground state by electric dipole selection rules ( $\Delta J=1$, yes). Such levels for most nuclei lie rather high in energy. It therefore is a reasonable approximation to assume that

$$
k \ll E_{b}-E_{c}, \quad k \ll E_{b^{\prime}}-E_{a},
$$

and replace the ratios $\left(E_{c}-E_{b}\right) /\left(E_{c}-E_{b}+k\right)$ and $\left(E_{a}-E_{b^{\prime}}\right) /\left(E_{a}-E_{b^{\prime}}-k\right)$ in eq. (3.3) by unity. Using this approximation and the completeness of the intermediate states, expression (3.3) becomes

$$
B_{j k}^{\prime}=\left\langle c\left|\left[\sum_{n=1}^{A}\left\{x_{k}\left(1+\tau_{3}\right)\right\}_{n}, \sum_{m=1}^{A}\left\{\sigma_{j} \tau_{+}\right\}_{m}\right]\right| a\right\rangle .
$$

In this commutator, only the isospin operators fail to commute, and for these we have the rule

$$
\left[\left\{\tau_{3}\right\}_{n},\left\{\tau_{+}\right\}_{m}\right]=2 \delta_{n m}\left\{\tau_{+}\right\}_{n}
$$

Hence, we have

$$
B_{j k}^{\prime}=2\left\langle c\left|\sum_{n=1}^{A}\left\{x_{k} \sigma_{j} \tau_{+}\right\}_{n}\right| a\right\rangle .
$$

The identity of expression (3.7) with expression (2.3) for the direct matrix element follows if we note that since the states $|a\rangle$ and $|c\rangle$ satisfy the unique first-forbidden selection rules $(\Delta J=2$, yes), only the symmetric traceless part of the operator in eq. (3.7) contributes to the matrix element.

We should stress that previous discussions of the effect of detour transitions have assumed that the only important contributions are from low-lying levels for which the energy denominators become small ${ }^{8,10}$ ). Nuclei for which this is the case are rare, but here we see that one can expect significant effects in all nuclei. Of course, if there is a low-lying level, one must correct for the error made in replacing the energy 
ratios in eq. (3.3) by unity. The largest effect would occur if there were a state $\tilde{b}$ in the daughter nucleus with an energy $E_{\tilde{b}}$ slightly higher than $E_{a}-m$. (For a lower energy, a regular allowed beta decay to $b$ would occur.) We would then write

$$
\frac{E_{\tilde{b}}-E_{c}}{E_{\tilde{b}}-E_{c}-k}=1+\frac{k}{E_{\tilde{b}}-E_{c}-k}
$$

and get

$$
B_{j k}^{\prime}=B_{j k}+\left\langle c\left|\sum_{n=1}^{A}\left\{x_{k}\left(1+\tau_{3}\right)\right\}_{n}\right| \tilde{b}\right\rangle \frac{k}{E_{\tilde{b}}^{\sim}-E_{c}-k}\left\langle\tilde{b}\left|\sum_{m=1}^{A}\left\{\sigma_{j} \tau_{+}\right\}_{m}\right| a\right\rangle .
$$

Such a correction might be important in some cases.

Finally, we would remark that the argument leading to the result given in eq. (3.3) is based upon a rather naive nuclear model namely that of a collection of $A$ Dirac nucleons interacting through a charge-independent scalar potential. Although one can argue that the result is more general than the model, we believe that the identity of the direct and detour nuclear matrix elements is only strongly indicated.

\section{Coulomb corrections}

For the direct decay, it was shown by Lewis and Ford that the effect of the Coulomb interaction to first order in $\alpha Z$ is to multiply the matrix elements by a factor of $1+$ $(\pi \alpha Z W / 2 \rho)$. One can easily extend their argument to show that the same factcr multiplies the detour matrix elements as well. The result therefore is that expression (2.7) for the beta-decay rate becomes

$$
\Gamma_{\beta}=\frac{g^{2} \lambda^{2} B_{r s} B_{r s}^{*}}{24 \pi^{3}} \int_{m}^{W_{0}} \mathrm{~d} W W p\left(W_{0}-W\right)^{2}\left[p^{2}+\left(W_{0}-W\right)^{2}\right]\left(1+\pi \alpha Z \frac{W}{p}\right) .
$$

Inserting the same factor in eq. (2.23) and using eq. (2.25), we get

$$
\begin{aligned}
\Gamma_{\gamma}= & \frac{\alpha g^{2} \lambda^{2} B_{r s} B_{r s}^{*}}{96 \pi^{5}} \int_{0}^{W_{0}-m} \mathrm{~d} k \int_{m}^{W_{0}-k} \mathrm{~d} W p W q^{2} k \sum_{\varepsilon=+1} \int \mathrm{d} \Omega\left(1+\pi \alpha Z \frac{W}{p}\right) \\
& \times\left\{\frac { p _ { 1 } ^ { 2 } + q ^ { 2 } } { W ( W _ { \mathrm { e } } ^ { 2 } - W _ { 1 } ^ { 2 } ) ^ { 2 } } \left[W\left(W_{0}^{2}+W_{1}^{2}\right)-2 W_{\bullet}\left(\hat{k} \cdot p \hat{k} \cdot p_{1}+m^{2}\right)\right.\right. \\
& \left.-2 \varepsilon k\left(W W_{\mathrm{e}}-m^{2}-\hat{k} \cdot p \hat{k} \cdot p_{1}\right)\right] \\
& +\frac{\xi}{W\left(W_{\mathrm{e}}^{2}-W_{1}^{2}\right)}\left[\left(2 W+\frac{1}{2} k\right)\left(p^{2}-\hat{k} \cdot p^{2}\right)-W k \cdot p_{1}+k p \cdot p_{1}\right. \\
& \left.-\frac{1}{2} \varepsilon k\left(p^{2}-\hat{k} \cdot p^{2}-2 W \hat{k} \cdot p_{1}+2 \hat{k} \cdot p \hat{k} \cdot p_{1}\right)\right] \\
& \left.+\eta\left[1-\frac{\varepsilon}{2 W} \hat{k} \cdot p\right]\right\}
\end{aligned}
$$

where $q=W_{0}-W-k$ and $\mathrm{d} \Omega$ is the solid angle of the photon with respect to the 
electron. In expression (4.2), we have introduced

$$
\begin{gathered}
\xi=\operatorname{R} 1\left\{B_{r s}^{*} B_{r s}^{\prime}\right\} / B_{r s} B_{r s}^{*}, \\
\eta=B_{r s}^{\prime} B_{r s}^{\prime *} / B_{r s} B_{r s}^{*} .
\end{gathered}
$$

If, as the discussion of sect. 3 implies, the nuclear matrix elements are the same for direct and detour transitions, then $\xi=\eta=1$.

\section{The spectrum, angular correlation and polarization}

From eq. (4.2) after summing over photon polarizations, we identify the differential rate in photon energy, electron energy and the angle between electron and photon. Dividing by eq. (4.1) and multiplying by the photen wave number, we obtain the following expression for the number of emissions per beta-decay differential in photon wave number, electron energy and the solid angle of the photon with respect to the electron:

$$
\begin{aligned}
& \frac{k}{\Gamma_{\beta}} \frac{\mathrm{d} \Gamma_{y}}{\mathrm{~d} k \mathrm{~d} W \mathrm{~d} \Omega}=\frac{\alpha}{\pi^{2} N\left(W_{0}\right)} p q^{2}\left(1+\pi \alpha Z \frac{W}{p}\right) \\
& \quad \times\left\{\frac{p_{1}^{2}+q^{2}}{4(W-k \cdot p)^{2}}\left[W\left(W_{e}^{2}+W_{1}^{2}\right)-2 W_{\mathrm{e}}\left(\boldsymbol{k} \cdot p k \cdot p_{1}+m^{2}\right)\right]\right. \\
& \left.\quad+\frac{\xi k}{2(W-k \cdot p)}\left[\left(2 W+\frac{1}{2} k\right)\left(p^{2}-k \cdot p^{2}\right)-W k \cdot p_{1}+k p \cdot p_{1}\right]+\eta k^{2} W\right\}
\end{aligned}
$$

where $q=W_{0}-k-W$ and

$$
N\left(W_{0}\right)=2 \int_{m}^{W_{0}} \mathrm{~d} W\left(W_{0}-W\right)^{2} W p\left[p^{2}+\left(W_{0}-W\right)^{2}\right]\left(1+\pi \alpha Z \frac{W}{p}\right) .
$$

Integrating eq. (5.1) over solid angles and electron energy, we obtain the following expression for the photon intensity per beta-decay differential in photon wave number

where

$$
\frac{k}{\Gamma_{\beta}} \frac{\mathrm{d} \Gamma_{\gamma}}{\mathrm{d} k}=\frac{2 x}{\pi N\left(W_{0}\right)}\left[F_{1}\left(k, W_{0}\right)+\xi F_{2}\left(k, W_{0}\right)+\eta F_{3}\left(k, W_{0}\right)\right],
$$

$$
\begin{array}{r}
F_{1}\left(k, W_{0}\right)=\int_{m}^{W_{0}-k} \mathrm{~d} W q^{2}\left(1+\pi \alpha Z \frac{W}{p}\right)\left\{\left[\left(W_{\mathrm{e}}^{2}+W^{2}\right)\left(W_{\mathrm{e}}^{2}-m^{2}+q^{2}\right)+2 m^{2} k W_{\mathrm{e}}\right]\right. \\
\left.\times \log \frac{W+p}{m}-2\left[W_{\mathrm{e}}\left(W_{\mathrm{e}}^{2}-m^{2}+q^{2}\right)+k\left(W_{\mathrm{e}}^{2}-W W_{\mathrm{e}}+W^{2}\right)\right] p\right\}, \\
F_{2}\left(k, W_{0}\right)=-k \int_{m}^{W_{0}-k} \mathrm{~d} W q^{2}\left(1+\pi \alpha Z \frac{W}{p}\right)\left\{m^{2}\left(2 W+\frac{3}{2} k\right) \log \frac{W+p}{m}\right. \\
\left.-\left(2 W^{2}+\frac{3}{2} k W-k^{2}\right) p\right\},
\end{array}
$$




$$
F_{3}\left(k, W_{0}\right)=2 k^{2} \int_{m}^{W_{0}-k} \mathrm{~d} W q^{2}\left(1+\pi \alpha Z \frac{W}{p}\right) W p .
$$

Similarly, we obtain the following expression for the photon polarization as a function of wave number:

$$
\begin{aligned}
P & \equiv \frac{\left\{\frac{\mathrm{d} \Gamma}{\mathrm{d} k}\right\}_{\mathrm{right}}-\left\{\frac{\mathrm{d} \Gamma}{\mathrm{d} k}\right\}_{\text {left }}}{\left\{\frac{\mathrm{d} \Gamma}{\mathrm{d} k}\right\}_{\mathrm{right}}+\left\{\frac{\mathrm{d} \Gamma}{\mathrm{d} k}\right\}_{\text {left }}} \\
& =-\frac{F_{4}\left(k, W_{0}\right)+\xi F_{5}\left(k, W_{0}\right)}{F_{1}\left(k, W_{0}\right)+\xi F_{2}\left(k, W_{0}\right)+\eta F_{3}\left(k, W_{0}\right)},
\end{aligned}
$$

where

$$
\begin{aligned}
& F_{4}\left(k, W_{0}\right)=k \int_{m}^{W_{0}-k} \mathrm{~d} W q^{2}\left(1+\pi \alpha Z \frac{W}{p}\right)\left\{\left[(2 W+k)\left(p^{2}+q^{2}+2 k W+k^{2}\right)+2 m^{2} k\right]\right. \\
& \left.\times \log \frac{W+p}{m}-2\left[p^{2}+q^{2}+3 k W+2 k^{2}\right] p\right\}, \\
& F_{5}\left(k, W_{0}\right)=-\frac{1}{2} k^{2} \int_{m}^{W_{0}-k} \mathrm{~d} W q^{2}\left(1+\pi \alpha Z \frac{W}{p}\right)\left[m^{2} \log \frac{W+p}{m}+(2 k-W) p\right] .
\end{aligned}
$$

These integrals are all expressed in terms of elementary functions in the appendix.

\section{Applications and comments}

To illustrate the magnitude of the effect of the detour transitions, we have calculated the spectrum and polarization for the decay of ${ }^{90} \mathrm{Y}\left(W_{0}=5.42 \mathrm{~m}\right)$. In fig. 2 , the spectrum is plotted for the case where the parameters $\xi=\eta=1$; for comparison the spectrum for the direct transition alone $(\xi=\eta=0)$ is also shown. The polarization is plotted in fig. 3 in the same manner. The correction to the spectrum is significant but not spectacular, the largest effects are found near the middle of the spectrum where the detour transitions increase the intensity by about $25 \%$. The effect of the detour transitions on the polarization is more striking but may be more difficult to observe.

We conclude with two comments.

(i) We have taken into account only the first-order Coulomb corrections, which for a nucleus like ${ }^{90} \mathrm{Y}\left(Z=40\right.$ in daughter $\left.{ }^{90} \mathrm{Z}\right)$ are not small. We do not know how to calculate correctly the higher-order corrections. Following the suggestion of Lewis and Ford ${ }^{11}$ ), one can attempt to take these higher-order corrections into account by replacing the factor $1+\pi x Z W / p$ by the Fermi function $F(Z, W)$. As shown by Lewis and Ford, this does not alter the first-order results significantly. 
(ii) We have calculated the effect of detour transitions only for unique first-forbidden beta decays. For allowed transitions $(\Delta J=0,1$, no), the detour transition must involve a combination of either a first-forbidden beta transition with electric dipole nuclear radiation or an allowed beta transition with magnetic dipole or electric quadrupole nuclear radiation. In either case, after performing the sums over all intermediate nuclear states, the nuclear matrix elements for the detour transition are of second-forbidden type and hence are entirely negligible. In the case of first-forbidden transitions other than unique, it should be clear that the discussion is valid just as in the case of the unique first-forbidden transition. The only difficulty is the

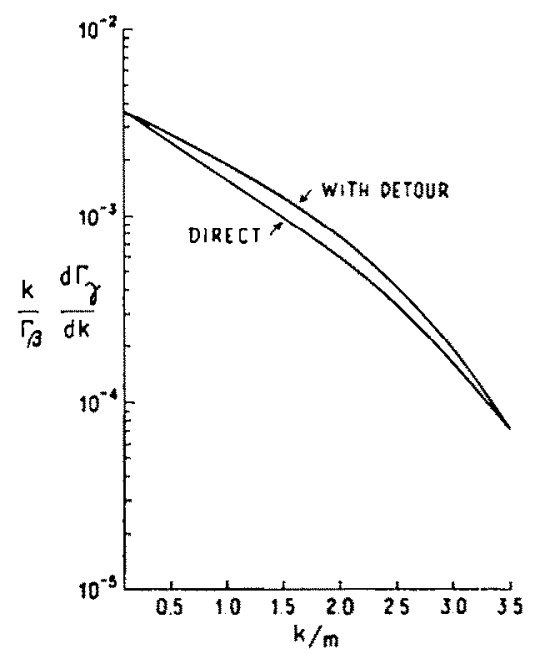

Fig. 2. The spectrum of inner bremsstrahlung for ${ }^{\circ} \mathrm{Y}$. The photon energy is in units of electron rest energy.

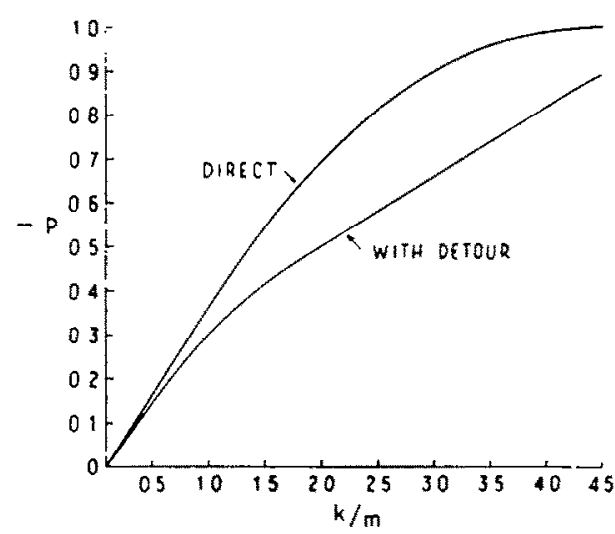

Fig. 3. The negative of the polarization of inner bremsstrahlung as function of photon energy for ${ }^{\circ} \mathrm{Y}$. The photon energy is in units of electron rest energy.

practical one that more than one nuclear matrix element appears, and these can be determined with only limited accuracy from the spectrum of the beta decay. Hence there are in effect additional parameters in the matrix elements for inner bremsstrahlung which make it difficult to identify experimentally the effects of the detour transitions. Of course if there is sufficient interest, the calculations can be performed for such cases as well as for second-forbidden decays.

Finally, we wish to acknowledge helpful conversations with $K$. T. Hecht, R. R. Lewis, Jr. and G. Parzen.

\section{Appendix}

The integrals occurring in sect. 5 are all elementary; we quote in this appendix the results of their evaluation. The first of these integrals may be expressed in terms of the 
integrals $I_{3}\left(x, W_{0}\right)$ and $I_{4}\left(x, W_{0}\right)$ of Lewis and Ford $\left.{ }^{11}\right)$. We find

$$
\begin{aligned}
& F_{1}\left(k, W_{0}\right)=m^{7}\left\{I_{3}\left(x, \frac{W_{0}}{m}\right)+\pi \alpha Z I_{4}\left(x, \frac{W_{0}}{m}\right)\right\}, \\
& F_{2}\left(k, W_{0}\right)=m^{7} y\left\{y^{2}\left[\left(\frac{1}{2} x^{2}+\frac{1}{8}\right) \log (x+s)-\left(\frac{1}{12} x^{2}+\frac{13}{24}\right) x s\right]\right. \\
& +y\left[-\left(\frac{1}{2} x^{2}+\frac{3}{8}\right) x \log (x+s)+\left(\frac{1}{20} x^{4}+\frac{83}{120} x^{2}+\frac{2}{15}\right) s\right] \\
& \left.+\left[-\left(\frac{1}{6} x^{4}-\frac{1}{4} x^{2}-\frac{1}{16}\right) \log (x+s)+\left(\frac{1}{30} x^{4}+\frac{41}{360} x^{2}-\frac{211}{720}\right) x s\right]\right\} \\
& +m^{7} \pi \alpha Z y\left\{-y^{2}\left[\frac{1}{12} x^{4}-\frac{1}{2} x^{2}+\frac{2}{3} x-\frac{1}{4}\right]\right. \\
& +y\left[\frac{3}{4} x \log ^{2}(x+s)-\left(\frac{1}{2} x^{2}+1\right) s \log (x+s)\right. \\
& \left.+\frac{1}{20} x^{5}+\frac{11}{12} x^{3}-2 x^{2}+\frac{5}{2} x-\frac{22}{15}\right] \\
& +\left[-\left(\frac{1}{2} x^{2}+\frac{3}{8}\right) \log ^{2}(x+s)-\left(\frac{1}{6} x^{2}-\frac{23}{12}\right) x s \log (x+s)\right. \\
& \left.\left.+\frac{1}{30} x^{6}+\frac{13}{72} x^{4}-\frac{79}{24} x^{2}+\frac{176}{43} x-\frac{5}{6}\right]\right\} \text {, } \\
& F_{3}\left(k, W_{0}\right)=m^{7} y^{2}\left[\frac{1}{2} x \log (x+s)+\left(\frac{1}{15} x^{4}-\frac{3}{10} x^{2}-\frac{4}{15}\right) s\right] \\
& +m^{7} \pi \alpha Z y^{2}\left[\frac{1}{15} x^{5}-\frac{2}{3} x^{2}+x-\frac{2}{5}\right] \text {, } \\
& F_{4}\left(k, W_{0}\right)=m^{7} y\left\{y^{3}\left[\left(\frac{1}{3} x^{2}+\frac{1}{2}\right) x \log (x+s)-\left(\frac{1}{18} x^{2}+\frac{2}{9}\right) s\right]\right. \\
& +y^{2}\left[\left(\frac{1}{3} x^{4}+x^{2}+\frac{1}{8}\right) \log (x+s)-\left(\frac{25}{36} x^{2}+\frac{55}{72}\right) x s\right] \\
& +y\left[\left(\frac{11}{30} x^{4}+\frac{4}{3} x^{2}+\frac{5}{16}\right) x \log (x+s)-\left(\frac{1417}{1800} x^{4}+\frac{4187}{3600} x^{2}+\frac{14}{223}\right) s\right] \\
& \left.+\left[\left(\frac{1}{10} x^{6}+\frac{1}{3} x^{4}-\frac{1}{16} x^{2}-\frac{1}{48}\right) \log (x+s)-\left(\frac{391}{1800} x^{4}+\frac{851}{3600} x^{2}-3 \frac{373}{600}\right) x s\right]\right\} \\
& +m^{7} \pi \alpha Z y\left\{y^{3}\left[-\frac{1}{2} x \log ^{2}(x+s)+\left(\frac{1}{3} x^{2}+\frac{2}{3}\right) s \log (x+s)-\frac{11}{18} x^{3}+x^{2}-\frac{7}{6} x+\frac{7}{9}\right]\right. \\
& +y^{2}\left[\left(x^{2}+\frac{3}{4}\right) \log ^{2}(x+s)+\left(\frac{1}{3} x^{2}-\frac{23}{6}\right) x s \log (x+s)-\frac{25}{36} x^{4}+\frac{91}{12} x^{2}-\frac{80}{9} x+2\right] \\
& +y\left[-\left(x^{2}+\frac{25}{8}\right) x \log ^{2}(x+s)+\left(\frac{11}{30} x^{4}+\frac{241}{60} x^{2}+\frac{58}{13}\right) s \log (x+s)\right. \\
& \left.-\frac{1417}{1800} x^{5}+x^{4}-\frac{2467}{360} x^{3}+\frac{104}{9} x^{2}-\frac{163}{15} x+\frac{1339}{225}\right] \\
& +\left[\left(\frac{1}{2} x^{4}+\frac{17}{8} x^{2}+\frac{1}{4}\right) \log ^{2}(x+s)+\left(\frac{1}{10} x^{4}-\frac{157}{60} x^{2}-\frac{97}{30}\right) x s \log (x+s)\right. \\
& \left.\left.-\frac{391}{1800} x^{6}+\frac{1949}{360} x^{4}-\frac{80}{9} x^{3}+\frac{539}{60} x^{2}-\frac{1328}{225} x+\frac{11}{18}\right]\right\} \text {, } \\
& F_{3}\left(k, W_{0}\right)=m^{7} y^{2}\left\{y\left[\left(\frac{1}{2} x^{2}+\frac{1}{8}\right) \log (x+s)-\left(\frac{1}{12} x^{2}+\frac{13}{24}\right) x s\right]\right. \\
& \left.+\left[-\left(\frac{1}{6} x^{2}+\frac{1}{8}\right) x \log (x+s)+\left(\frac{1}{60} x^{4}+\frac{83}{360} x^{2}+\frac{2}{43}\right) s\right]\right\} \\
& +m^{7} \pi \alpha Z y^{2}\left\{y\left[-\frac{1}{12} x^{4}+\frac{1}{2} x^{2}-\frac{2}{3} x+\frac{1}{4}\right]\right. \\
& +\left[\frac{1}{1} x \log ^{2}(x+s)-\left(\frac{1}{6} x^{2}+\frac{1}{3}\right) s \log (x+s)\right. \\
& \left.\left.+\frac{1}{60} x^{5}+\frac{11}{36} x^{3}-\frac{2}{3} x^{2}+\frac{5}{6} x-\frac{22}{45}\right]\right\} \text {. }
\end{aligned}
$$

In these expressions

$$
x=\frac{W_{0}-k}{m}, \quad y=\frac{k}{m}, \quad s=\left(x^{2}-1\right)^{t} .
$$




\section{References}

1) W. Kreische, W. Lampert and G. Loos, Nucl. Phys. A107 (1968) 601

2) B. Persson, Nucl. Phys. 67 (1965) 121

3) B. Persson, Nucl. Phys. 55 (1964) 49

4) J. K. Knipp and G. E. Uhlenbeck, Physica 3 (1936) 425

5) F. Block, Phys. Rev. 50 (1936) 272

6) D. Gebhardt, Nucl. Phys. A107 (1968) 593

7) D. Gebhardt, Nucl. Phys. A115 (1968) 207

8) C. L. Longmirc, Phys. Rev. 75 (1949) 15

9) J. Horowitz, J. Phys. Rad. 13 (1952) 429

10) M. E. Rose, L. L. Foldy and R. Perrin, Phys. Rev. 128 (1962) 1776

11) R. R. Lewis, Jr., and G. W. Ford, Phys. Rev. 107 (1957) 756 